\title{
Photonic integrated circuit on InP for millimeter wave generation
}

\author{
Frederic van Dijk ${ }^{1}$, Marco Lamponi ${ }^{1}$, Mourad Chtioui ${ }^{2}$, François Lelarge ${ }^{1}$, Gaël Kervella ${ }^{1}$, \\ Efthymios Rouvalis ${ }^{3}$, Cyril Renaud ${ }^{3}$, Martyn Fice $^{3}$, Guillermo Carpintero ${ }^{4}$ \\ ${ }^{1}$ III-V Lab, a joint Laboratory of "Alcatel Lucent Bell Labs", "Thales Research \& Technology" and \\ "CEA-LETI", Palaiseau, France (e-mail : frederic.vandijk@3-5lab.fr), ${ }^{2}$ Thales Air Systems, 91470 \\ Limours, France, ${ }^{3}$ Department of Electronic and Electrical Engineering, UCL, Torrington Place, \\ WC1E 7JE, United Kingdom, ${ }^{4}$ Universidad Carlos III de Madrid, Av de la Universidad, 30. \\ Leganes 28911 Madrid, Spain
}

\begin{abstract}
Indium phosphide and associated epitaxially grown alloys is a material system of choice to make photonic integrated circuits for microwave to terahertz signal generation, processing and detection. Fabrication of laser emitters, high speed electro-optical modulators, passive waveguides and couplers, optical filters and high speed photodetectors is well mastered for discrete devices. But monolithic integration of them while maintaining good performances is a big challenge. We have demonstrated a fully integrated tunable heterodyne source designed for the generation and modulation of sub-Terahertz signals. This device is to be used for high data-rate wireless transmissions. DFB lasers, SOA amplifiers, passive waveguides, beam combiners, electro-optic modulators and high speed photodetectors have been integrated on the same InP-based platform. Millimeter wave generation at up to $120 \mathrm{GHz}$ based on heterodyning the optical tones from two integrated lasers in an also integrated high bandwidth photodetector has been obtained.
\end{abstract}

Keywords: millimeter wave generation; semiconductor lasers; photonic integrated circuits

\section{INTRODUCTION}

Systems suitable for the generation and transmission of millimeter wave frequencies $(30 \mathrm{GHz}-300 \mathrm{GHz})$ are of great interest in the development of broadband wireless communication systems [1]. Millimeter-wave photonic devices capable of generate carrier frequencies from $30 \mathrm{GHz}$ to $200 \mathrm{GHz}$ have already been demonstrated [2-4]. Some of the proposed solutions involved discrete devices associated with free space optics [5]. More recently hybrid integration has been employed in order to reduce the packaging size and optical delays inside the chip [6]. In the long term, monolithic integration promises to allow cost effective production, even tighter packaging, and fewer alignment issues [7-8]. However the challenge of monolithic integration is to combine the needed high performances of two tunable lasers with a high bandwidth photodiode on the same substrate.

In our previous published works high bandwidth Uni-Traveling Carrier Photodiodes (UTC-PDs) compatible with active/passive integration were demonstrated [2]. We have also demonstrated monolithic integration of two narrow linewidth DFB lasers with passive optical waveguides and couplers [4]. In this paper we demonstrate active/passive integration that allows Distributed Feedback (DFB) Lasers, Semiconductor Optical Amplifiers (SOAs), electro-optical modulators, Multimode Interference (MMI) couplers and high speed UTC-PDs on the same chip.

\section{DEVICE DESCRIPTION}

Fig. 1 shows the device that has been characterized for the results presented in this paper. It is composed of two $1 \mathrm{~mm}$ long DFB lasers with a phase shift in the middle of the Bragg grating in order to guarantee single mode operation. The outputs from the two lasers are combined, after passing through bent SOAs, using a multimode interference (MMI) coupler. Each output of the coupler is evanescently coupled to an UTC photodiode after passing through two SOAs and one electro-optical modulator. On the other side of the chip, light from the DFB lasers is combined in a MMI coupler in order to have an optical access to the chip. The whole device is $4.4 \mathrm{~mm}$ long and $0.7 \mathrm{~mm}$ wide.

Integrated Optics: Devices, Materials, and Technologies XVIII, edited by Jean Emmanuel Broquin, Gualtiero Nunzi Conti, Proc. of SPIE Vol. 8988, 89880Q · (c) 2014 SPIE

CCC code: $0277-786 X / 14 / \$ 18 \cdot$ doi: $10.1117 / 12.2036571$ 


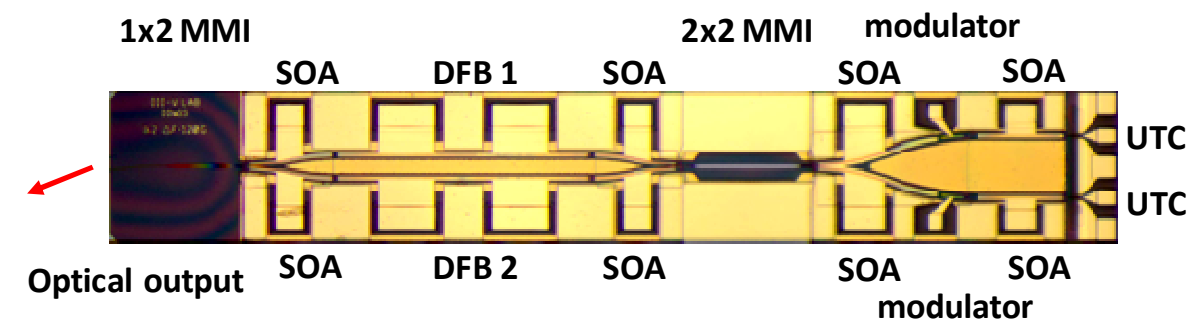

Figure 1. microscope view of one chip.

In these devices each DFB laser generates an optical tone from which the output power can be adjusted using the independently electrically connected SOAs. The two optical tones are sent to the high speed UTC photodiode in which the optical signal is converted into a high speed electrical signal at a frequency equal to the difference between the two optical frequencies.

\section{DEVICE FABRICATION}

The layers were grown on a semi-insulating InP wafer in order to reduce the parasitic capacitance and get a sufficiently large detection bandwidth of the photodiodes. Active/passive integration is achieved using a butt-joint process. The active layers consist of 6 InGaAsP quantum wells. DFB lasers, SOAs and modulation sections contain the same quantum well stack. The Bragg grating is formed in a InGaAsP layer placed above the quantum wells and defined by e-beam lithography. The UTC layers, that are similar to the layers used in [2], are grown above the passive waveguide. The fabrication needed 3 epitaxial growth steps. After wafer thinning and back metal deposition chips were cleaved and mounted on AlN submounts.

\section{OPTICAL AND ELECTRICAL BEAT-NOTE MEASUREMENTS}

One of the devices was characterized in terms of optical spectrum and generated high frequency tone. For these measurements we used the setup presented in Fig. 2. A multi-DC probe is used in order to apply the bias currents to the different sections. One of the two photodiodes is probed using an ACP 110 coplanar probe from Cascade Microtech and biased through an SHF BT110 bias tee at $-3.5 \mathrm{~V}$. An ACP 65 coplanar probe is used to probe the electro-optical modulator section. A lensed fibre is used to retrieve the optical signal from the back of the chip.

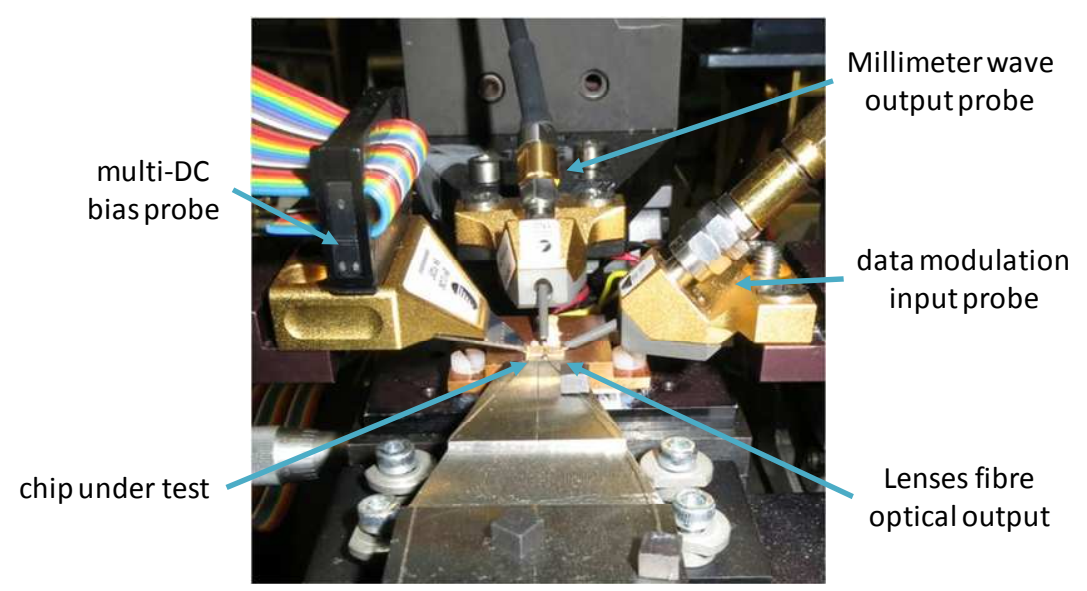

Figure 2. general view of the test setup

We have measured simultaneously the spectrum of the optical signal generated by the chip and the generated high frequency electrical signal from the monolithically integrated UTC photodiodes when the DFB lasers were electrically tuned and some of the SOAs biased. 


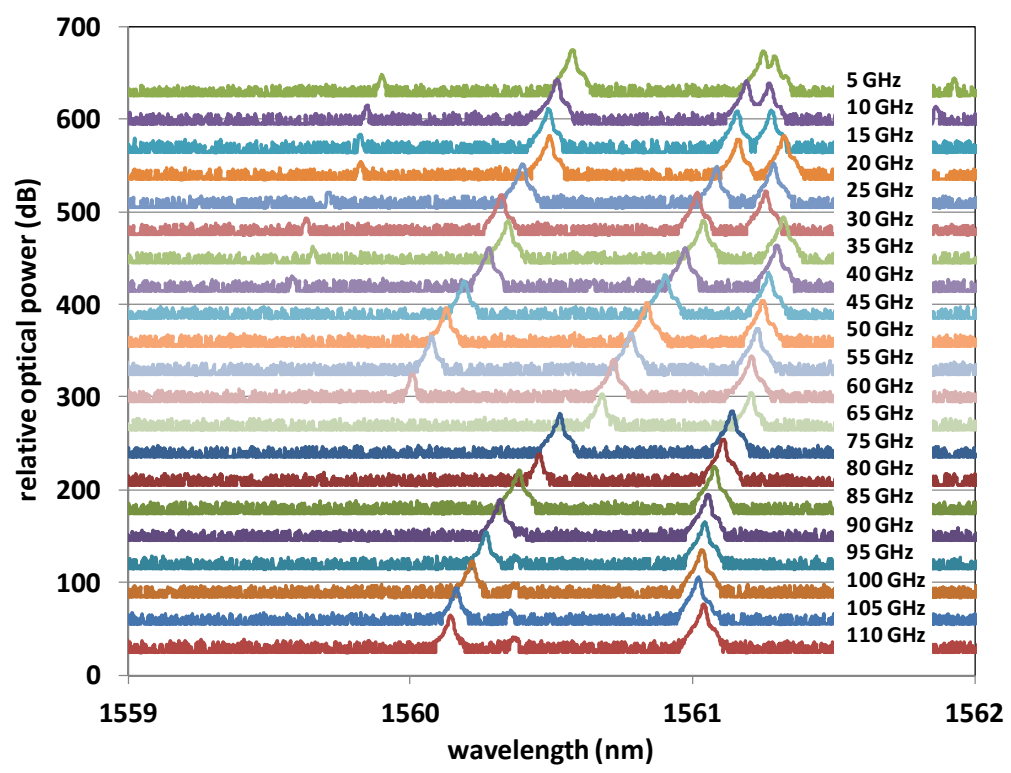

Figure 3. optical spectra for different bias currents

For these measurements, one DFB laser was biased with a current varying within a 50 to $86 \mathrm{~mA}$ range and the other was biased biased with a current varying within a 50 to $198 \mathrm{~mA}$ range. Within this bias range the UTC photocurrent was between 1.12 and $6.27 \mathrm{~mA}$. The measured optical spectra are presented in Fig. 3. With this device we obtain a continuous tuning of the optical frequency difference between the two DFB tones from 5 to $110 \mathrm{GHz}$. The wavelength tuning is thermal. As the two lasers are close from each-other, wavelength of DFB2 changes also due to thermal crosstalk. For the upper curves on graph $(21-50 \mathrm{GHz})$, there is an additional optical tone. This is due to the tendency of each DFB lasers to have a dual mode behavior for higher bias currents. Indeed the DFB lasers, with an integrated phase shift, are single mode in the ideal case, where there are no reflections except those due to the Bragg gratings. In the integrated chip there are some residual reflections that can occur, for example, on the butt joints, in the MMI coupler or on the back of the photodiodes.

The electrical spectra are measured using a FSU67 Rohde\&Schwarz electrical spectrum analyzer. For measurements above $65 \mathrm{GHz}$, a Rohde\&Schwartz FS-Z110 external mixer is used in combination with the same coplanar probe and bias tee. The measurements are presented in Fig. 4. 


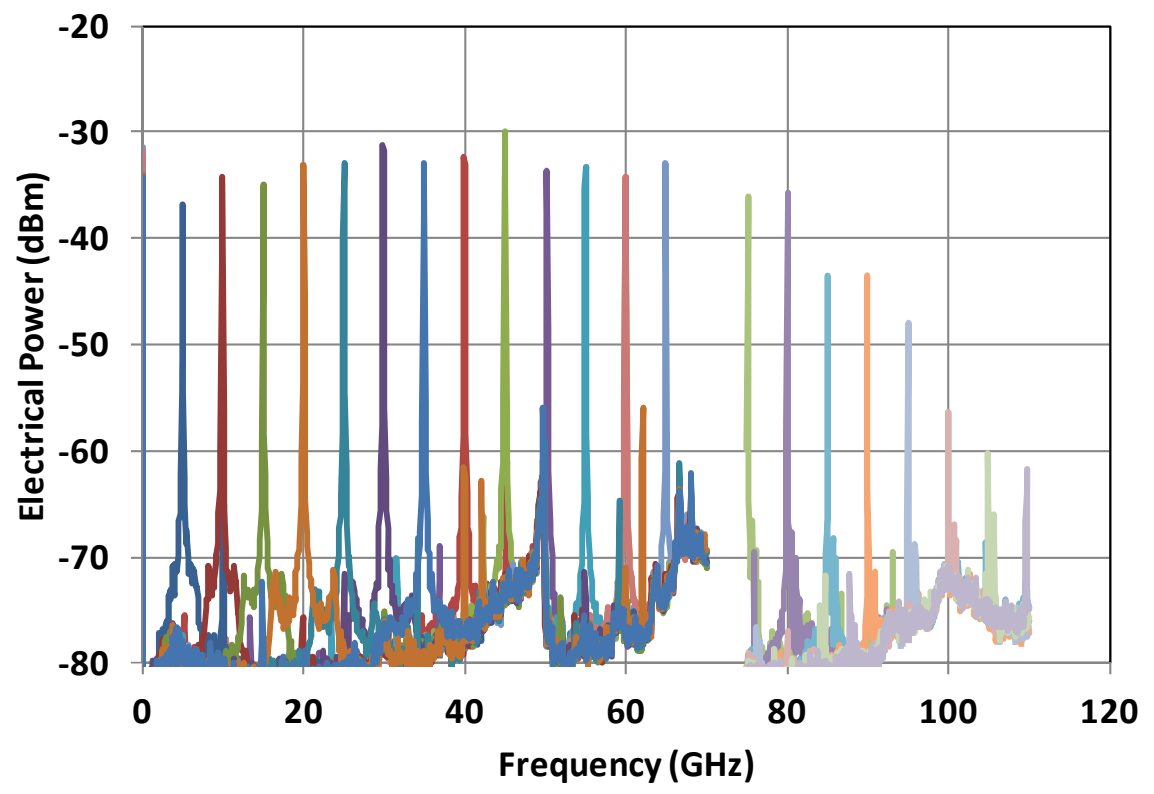

Figure 4. electrical spectra obtained for different laser biasing conditions

We were able to observe the beat note on the photodiode port over the full tuning range. In order to have a better idea of the bandwidth of the integrated photodiodes, we have corrected the power level of the detected high frequency tones by subtracting the losses of the cables and the probes and by normalizing it based on the measured photocurrent. The result is presented in Fig. 5.

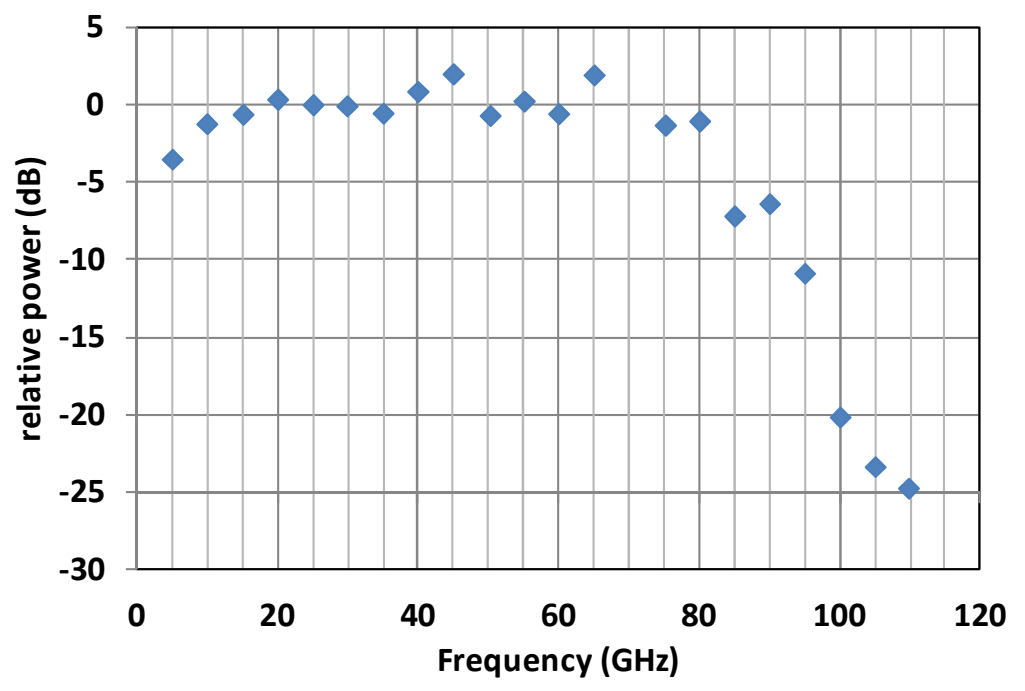

Figure 5. evaluated relative high frequency response of the integrated photodiode

From these measurements it seems that the bandwidth of the photodiode is larger than $80 \mathrm{GHz}$. Complementary measurements of the bandwidth on discrete devices cleaved from the wafers need to be done in order to have a more precise idea of the actual bandwidth of these photodiodes.

\section{WIRELESS DATA TRANSMISSION}

We have made very short distance wireless transmission (less than one centimeter) to validate the functionality of the device. For this DFB1 and DFB2 were biased respectively at 172 and $126 \mathrm{~mA}$ in order to get a beat note at $90 \mathrm{GHz}$. The 
signal from the PRBS generator is sent to the modulation section of the chip through a bias tee. The modulator is biased at $8 \mathrm{~mA}$. SOA sections between the DFB lasers and the $2 \times 2$ coupler are biased with the same supply at $190 \mathrm{~mA}$. The two SOAs before and after the modulator are biased with the same supply at $200 \mathrm{~mA}$. The photocurrent from the $-3.5 \mathrm{~V}$ biased photodiode is $3.04 \mathrm{~mA}$. After it is photo-detected in the chip, the modulated millimeter signal is sent to the horn antenna, retrieved by a second horn antenna, down converted using an harmonic mixer, amplified, rectified using a crystal diode detector, filtered and sent to a sampling oscilloscope. For these experiments the distance between the two antennas was very limited, about two millimeters. Fig. 6 shows the elements of the setup that was built around the unpackaged chip. Fig. 7 shows the retrieved signal for a $100 \mathrm{MBit} / \mathrm{s}$ signal.

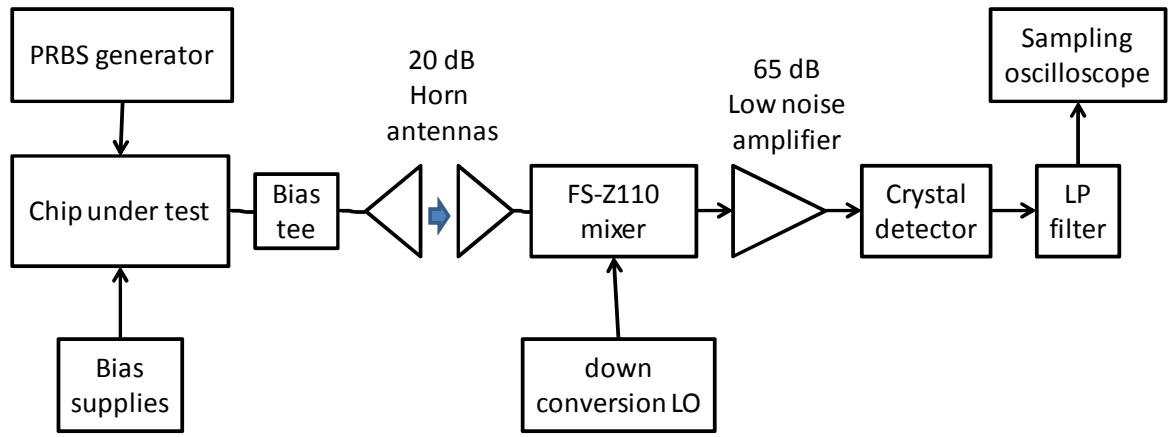

Figure 6. wireless data transmission experiment diagram

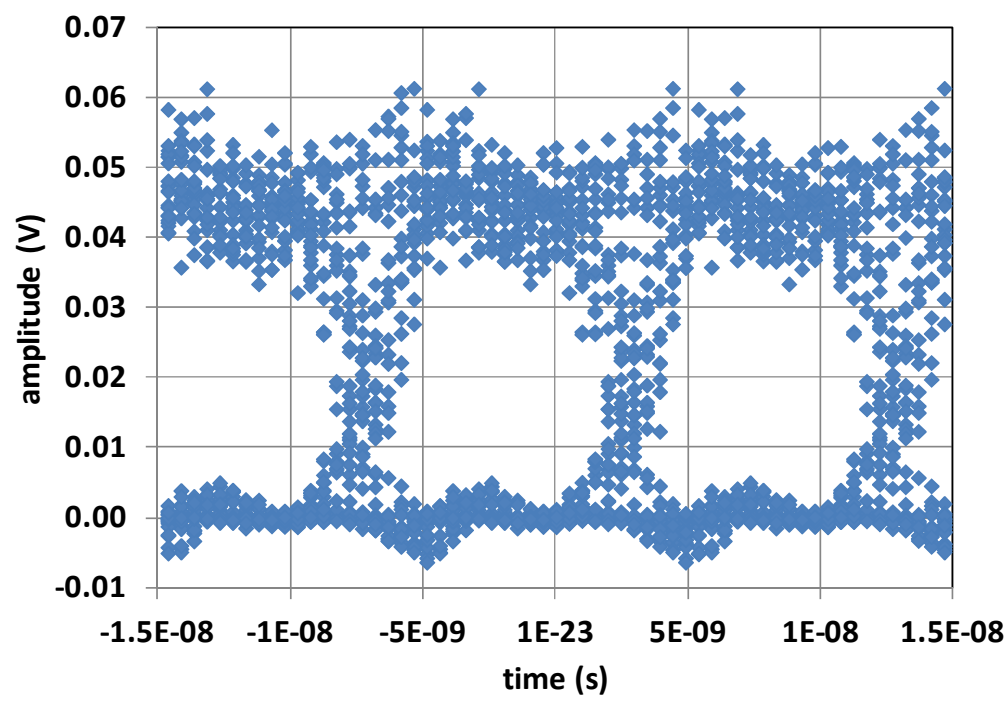

Figure 7. reconstructed eye diagram for $100 \mathrm{MBit} / \mathrm{s}$ transmission

We can see that the transmitted signal presents a clear open eye compatible with a good data transmission. Transmissions at higher data rates and for longer distances are currently being tested. 


\section{CONCLUSIONS}

We have demonstrated the integration of a widely tunable heterodyne system that associates DFB lasers, SOAs, passive waveguides, electro-optical modulators, optical couplers and UTC-type high bandwidth photodetectors in the same InP based chip.

Optical measurements have shown that the devices has a tuning range of the optical frequency difference from 5 to 110 GHz. Measurements of the heterodyne beat note generated on a device show the ability to emit a beat note at up to 110 $\mathrm{GHz}$. A preliminary evaluation of the bandwidth of the UTC photodiode make us expect it to exceed $80 \mathrm{GHz}$. The device has been successfully used in at $100 \mathrm{MBit} / \mathrm{s}$ data over a $90 \mathrm{GHz}$ carrier wireless transmission.

\section{ACKNOWLEDGEMENTS}

The authors acknowledge cooperation within and financial support by the EC projects iPHOS and IPHOBAC-NG.

\section{REFERENCES}

[1] Stöhr, A., "Photonic Millimeter-Wave Generation and its Applications in High Data Rate Wireless Access," Proc. IEEE International Topical Meeting on Microwave Photonics, (2010).

[2] Rouvalis, E. ; Cthioui, M. ; van Dijk, F. ; Fice, M.J. ; Carpintero, G. ; Renaud, C.C. ; Seeds, A.J, "170 GHz Photodiodes for InP-based photonic integrated circuits," Proc. IEEE Photonics Conference, (2012)

[3] Ristic, S.; Bhardwaj, A.; Rodwell, M.J.; Coldren, L.A.; Johansson, L.A.; "An Optical Phase-Locked Loop Photonic Integrated Circuit," IEEE Journal of Lightwave Technology, Volume: 28 , Issue: 4 , 526 - 538 (2010)

[4] van Dijk, F. ; Accard, A. ; Enard, A. ; Drisse, O. ; Make, D. ; Lelarge, F., "Monolithic dual wavelength DFB lasers for narrow linewidth heterodyne beat-note generation," Proc. IEEE International Topical Meeting on Microwave Photonics, 73 - 76 (2011)

[5] L. N. Langley, M.D. Elkin, C. Edge, M. J. Wale, U. Gilese, X. Huang, A. J. Seeds, "Packaged semiconductor laser optical phase-locked loop (OPLL) for photonic generation, processing and transmission of microwave signals," IEEE trans. on Microw. Theory and Techn. 47(7), 1257-1264 (1999). 